\title{
SARS-CoV uses ACE2 and CD209L receptors in infection
}

\begin{abstract}
Diabetes causes an increase in the expression of Angiotensin-converting enzyme 2 (ACE 2). In fact, the receptor ACE2 is a portal of entry of SARS-CoV. in this discussion, we focus on another receiver, CD209L, which also facilitates entry of SARS-CoV into the cell in diabetic subjects. (Clin Diabetol 2021; 10; 3: 243-244)
\end{abstract}

Key words: COVID-19, diabetes, infection, immune system

Diabetes is a rapidly growing public health problem, and it is a well-known risk factor for infectious disease because it weakens the immune system.

In healthy subjects, acute hyperglycemia has been shown to reduce neutrophil degranulation.

Natural killer (NK) cells play a crucial role in defending the body against infections and tumors. These cells have the ability to recognize and treat tumors and viral or bacterial infections. Their activity is regulated by activating receptors, including NKp30, NKp44, NKp46, NKG2C and NKG2D, which all bind ligands present at the surface of tumors or infected cells; NKp46 is a specific NK receptor able to recognize various influenza hemagglutinins, and NKG2D is expressed on NK cells, CD8-positive TCRab T cells, and CD8-positive TCRcdT cells [1].

Address for correspondence:

Fatima Rachidi

Biology Department

Higher School of Teachers (ENS)

Mohammed V University, Rabat, Morocco

e-mail: fatimarachidi1@yahoo.fr

Clinical Diabetology 2021, 10; 3: 243-244

DOI: 10.5603/DK.a2021.0017

Received: 26.01.2021

Accepted: 29.11.2021
Diabetes alters NK cell phenotype and function; in fact, patients with type 2 diabetes have an abnormal NK cell phenotype with a significant decrease in frequency of both NKp46- and NKG2D-positive NK cells, in addition to functional defects of the NK cell population, which showed reduced degranulation [1].

SARS-CoV uses his crucial receptor ACE2 (angiotensin-converting enzyme 2) as a portal of entry [2]. But there is a second receptor, CD209L, a C-type lectin family (also called L-SIGN, DCSIGNR, and DC-SIGN2), which has been identified as a functional SARS coronavirus receptor in cell lines, and it can also serve as a portal of entry for infectious SARS-CoV $[3,4]$.

Both SARS-CoV receptors of ACE2 and CD209L are expressed in the organ/tissue-derived endothelial cells in human. The expression of ACE2 receptor is the highest in the human lung microvascular endothelial cells, and lowest in the lymphatic endothelial cells [3]. CD209L is expressed in human lung in type II alveolar cells and endothelial cells, both targets for SARS-CoV [4], but it is relatively higher in the human lymphatic endothelial cells [3].

Coronaviruses bind to their glycoprotein receptors by the 200-kDa spike glycoproteins, S, which are some of the largest viral spike glycoproteins. Moreover, it is possible that different domains within a single $\mathrm{S}$ protein could recognize multiple alternative receptors. In addition, the large $S$ glycoprotein of SARS-CoV uses both ACE2 and CD209L in virus infection and pathogenesis $[3,4]$.

The effect of acute hyperglycemia on ACE2 expression which might facilitate viral cell entry is well documented [2]. Furthermore, diabetes disrupts C-type lectin function. Indeed, within the immune system, host-pathogen and regulatory host-host interactions operate through binding of oligosaccharides by C-type lectin; hyperglycemia affects protein-oligosaccharide interactions via competitive inhibition [5]. 


\section{Acknowledgments}

This work is supported by Mohammed V University.

\section{Conflict of interest}

I declare no competing interest.

\section{REFERENCES}

1. Berrou J, Fougeray $S$, Venot $M$, et al. Natural killer cell function, an important target for infection and tumor protection, is impaired in type 2 diabetes. PLoS One. 2013; 8(4): e62418, doi: 10.1371/ journal.pone.0062418, indexed in Pubmed: 23638076.

2. Bindom SM, Lazartigues $E$. The sweeter side of ACE2: physiological evidence for a role in diabetes. Mol Cell Endocrinol. 2009;
302(2): 193-202, doi: 10.1016/j.mce.2008.09.020, indexed in Pubmed: 18948167.

3. Li J, Gao J, Xu YP, et al. Zhonghua Yi XueZaZhi. Expression of severe acute respiratory syndrome coronavirus receptors, ACE2 and CD209L in different organ derived microvascular endothelial. 2007; 87(12): 833-837.

4. Jeffers SA, Tusell SM, Gillim-Ross L, et al. CD209L (L-SIGN) is a receptor for severe acute respiratory syndrome coronavirus. Proc Natl Acad Sci U S A. 2004; 101(44): 15748-15753, doi: 10.1073/ pnas.0403812101, indexed in Pubmed: 15496474.

5. Ilyas R, Wallis R, Soilleux EJ, et al. High glucose disrupts oligosaccharide recognition function via competitive inhibition: a potential mechanism for immune dysregulation in diabetes mellitus. Immunobiology. 2011; 216(1-2): 126-131, doi: 10.1016/j. imbio.2010.06.002, indexed in Pubmed: 20674073. 